\title{
Efficacy of Carboxymethylcellulose and Hyaluronate in Dry Eye Disease: A Systematic Review and Meta-Analysis
}

\author{
Jae Kyeong Song', Kiheon Lee ${ }^{2, *}$, Hwa Yeon Park', Joon Young Hyon ${ }^{3}$, Seung-Won $\mathrm{Oh}^{4}$, Woo Kyung Bae ${ }^{5}$, \\ Jong-Soo Han ${ }^{5}$, Se Young Jung' ${ }^{2}$, Yoo Jin Um², Ga-Hye Lee', Ji Hye Yang' \\ 'Department of Family Medicine, Seoul National University Hospital, Seoul, Korea \\ 2Department of Family Medicine, Seoul National University Bundang Hospital, Seoul National University College of Medicine, Seongnam, Korea \\ ${ }^{3}$ Department of Ophthalmology, Seoul National University Bundang Hospital, Seoul National University College of Medicine, Seongnam, Korea \\ ${ }^{4}$ Department of Family Medicine, Healthcare System Gangnam Center, Seoul National University Hospital, Seoul, Korea \\ ${ }^{5}$ Department of Family Medicine, Health Promotion Center, Seoul National University Bundang Hospital, Seongnam, Korea
}

Background: The efficacy of two artificial tears, carboxymethylcellulose (CMC) and hyaluronate (HA), was compared in the treatment of patients with dry eye disease.

Methods: We conducted a systematic review and meta-analysis on randomized controlled trials in the PubMed, Embase, Cochrane Library, and ClinicalTrials.gov databases. The efficacy was compared in terms of the mean change from baseline in tear break-up time. The meta-analysis was conducted using both random and fixed effect models. The quality of the selected studies was assessed for risk of bias.

Results: Five studies were included involving 251 participants. Random effect model meta-analysis showed no significant difference between CMC and HA in treating dry eye disease (pooled standardized mean difference [SMD]=-0.452; 95\% confidence interval [CI], -0.911 to $0.007 ; \mathrm{P}=0.053$ ). In contrast, fixed effect model meta-analysis revealed significant improvements in the CMC group when compared to the HA group (pooled SMD $=-0.334 ; 95 \%$ CI, -0.588 to $-0.081 ; \mathrm{P}=0.010$ ).

Conclusion: The efficacy of $\mathrm{CMC}$ appeared to be better than that of HA in treating dry eye disease, although metaanalysis results were not statistically significant. Further research is needed to better elucidate the difference in efficacy between $\mathrm{CMC}$ and $\mathrm{HA}$ in treating dry eye disease.

Keywords: Xerophthalmia; Carboxymethylcellulose Sodium; Hyaluronate; Fluorescein Tear Breakup Time 


\section{INTRODUCTION}

Dry eye is a disorder involving deficient tear production or excessive tear evaporation, ${ }^{1)}$ and is associated with subjective symptoms, such as ocular discomfort, dryness, visual disturbance, and soreness. ${ }^{2,3)} \mathrm{Ob}$ jective signs include ocular surface inflammation, tear film instability, and tear hyperosmolarity. ${ }^{3)}$ Dry eye is a very common disorder, reported in approximately $10 \%-20 \%$ of the adult population. ${ }^{4)}$

Artificial tears are the primary treatment for dry eye disease. ${ }^{5)} \mathrm{Al}$ though there are many effective artificial tear formulations, carboxymethylcellulose (CMC) and hyaluronate (HA) are the two most commonly prescribed and used. ${ }^{6)} \mathrm{CMC}$ is an anionic cellulose polymer with a carboxyl group substitution, and exhibits good bioadhesive characteristics. ${ }^{7,8)}$ The anionic nature of CMC may be beneficial in increasing tear retention time ${ }^{6}$ HA is a glycosaminoglycan disaccharide biopolymer composed of repeating alternating sequences of $\mathrm{N}$-acetylglucosamine and glucuronate in linear chains; importantly, HA formulations have the ability to bind water molecules and prevent dehydration. ${ }^{9,10)}$

Large clinical studies have shown both CMC and HA to improve dry eye signs and symptoms. In an effort to identify the most effective treatment, several studies have compared the efficacies of CMC and HA by various methods including tear break-up time (TBUT), corneal or conjunctival staining, Schirmer test, and dry eye symptom score. ${ }^{6,11-18)}$ While some studies have reported that CMC and HA have an equal effect in treating dry eye disease, other studies have showed that one treatment choice was more effective than the other. Due to the contrasting results, and in an attempt to identify the most effective treatment, we decided to conduct a systematic review and meta-analysis aimed at comparing the efficacy of CMC and HA.

\section{METHODS}

This study followed the PRISMA statement for reporting systematic reviews and meta-analysis of studies that evaluate health care interventions. ${ }^{19)}$

\section{Inclusion Criteria}

We included randomized clinical trials (RCTs) which compared the efficacy of CMC and HA in humans with dry eye disease. Due to the fact that there are several tests to evaluate the degree of dry eye disease, and that their scale or index was inconsistent across studies, we decided to use TBUT test results to compare studies. TBUT should be measured as the time from last blink to the appearance of the dry spot in the precorneal tear film, and only studies that measured TBUT at both baseline and at follow-up were selected.

\section{Search Strategy}

We searched Pubmed, Embase, Cochrane Library, and ClinicalTrials. gov using the following intervention terms "((hyaluron*) OR (vismed OR kynex OR lubristil)) AND ((carboxymethyl*) OR (cellulose gum) OR (carmel*) OR (optive OR refresh plus OR viscofresh OR cellufresh OR celluvisc))" and patient terms "((dry eye) OR (keratoconjunctivitis sicca OR sjogren syndrome OR xerophthalmia))," including MeSH

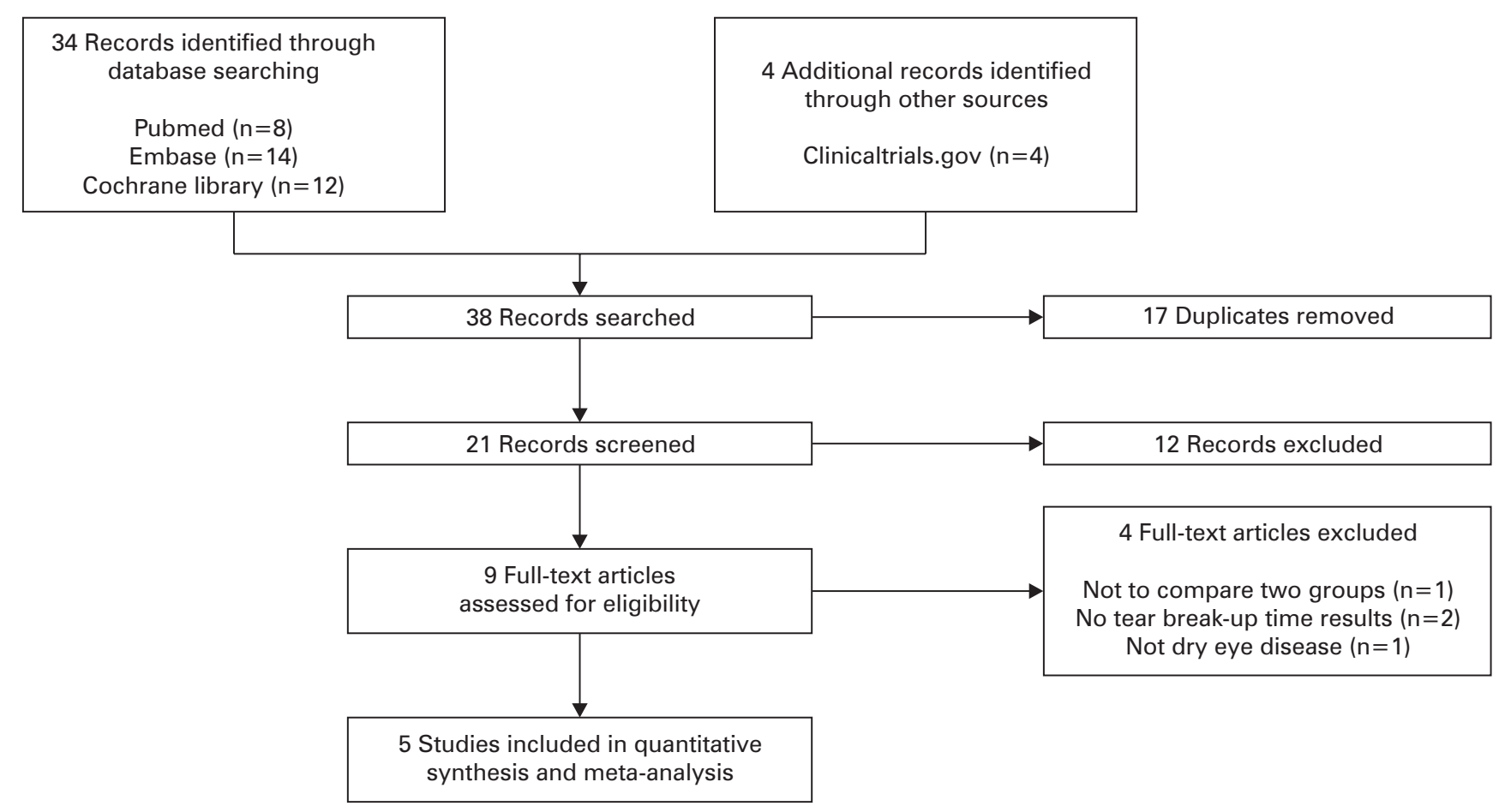

Figure 1. Study flow chart. 
terms. The databases were last searched on December 20, 2015 with no publication date limitation and no language restriction. Further search conditions restricted studies to those conducted on humans using RCT or controlled clinical trial study designs.

\section{Study Selection}

Figure 1 presents the search results and study selection. Two review authors (JKS and HYP) independently screened all titles and abstracts obtained from the search. The combined search revealed 38 records, from which 17 records were removed as duplicates. Titles and abstracts were reviewed for the remaining records, from which 12 were excluded and nine full-text articles were reviewed against the inclusion criteria. Four articles were excluded for the following reasons: one article was not designed to compare two groups, ${ }^{14)}$ two articles had no TBUT data, ${ }^{16,18)}$ and, while the final article had TBUT data, the baseline value was above 10 seconds in the HA group, which did not meet the criteria for dry eye disease diagnosis. ${ }^{12)}$ The remaining five studies were included in quantitative synthesis and meta-analysis. ${ }^{6,11,13,15,17)}$ Any disagreement was resolved by discussion with a third reviewer (KL). This study was exempted from review by the institutional review board of Seoul National University Bundang Hospital.

\section{Data Extraction and Analysis}

Effect size, which was represented by mean TBUT change from baseline, and its standard deviation was extracted from each of the selected studies. The standard deviation of the effect size was not reported in two studies, namely Sanchez et al. ${ }^{15)}$ study in 2010 and Brignole et al. ${ }^{13)}$ study in 2005. We contacted the authors, but received no reply. The missing values were estimated by pooling the standard deviation using the Hedges method. ${ }^{20)}$ We performed Hedges random and fixed effect model meta-analyses using the 'METAN' package in Stata (Stata Co., College Station, TX, USA). The significance level was defined as a Pvalue less than 0.05 . We also assessed heterogeneity using Higgin's $I^{2}$ statistic, where a heterogeneity $\left(\mathrm{I}^{2}\right)$ of more than $50 \%$ was defined as high.

\section{Quality Assessment}

The quality of the studies included in the meta-analysis was assessed by the approach recommended in the Cochrane handbook for systematic reviews of interventions. ${ }^{21)}$ We assigned a grade (low, high, or unclear) for risk of bias for seven domains.

\section{RESULTS}

\section{Characteristics of Included Studies}

Table 1 presents summarized data of the included studies. All five studies were RCTs and the combined sample size was 251 participants. The studies did not show a statistically significant difference between the characteristics of the study populations of the two treatment groups; the only exception was for age in the paper published by Sanchez et al. ${ }^{15)}$ in 2010. In the study, mean age of the HA group was 71.8

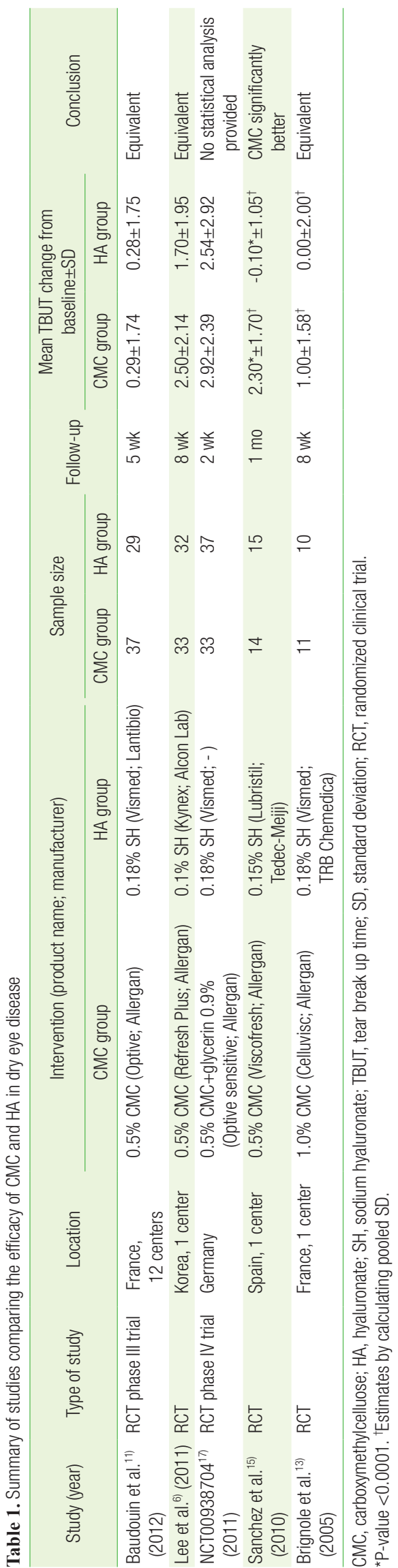


years, while mean age of the CMC group was 51.8 years (P-value $<0.005)$.

Because commercially available CMC and HA eye drop products were used, there was some variation in the concentrations of the active ingredients across the studies. The recommended dosage for both products was one drop, three to six times a day. The length of followup varied from 2 to 8 weeks.

The mean TBUT changes from baseline to the primary end point in CMC and HA groups were extracted from the studies. The effect size was calculated as the standardized mean difference (SMD) between the two groups. One study revealed an almost equal efficacy between the two groups (Baudouin et al. ${ }^{11)}$ study in 2012, SMD=-0.006). The effect sizes of three studies favored CMC but were not statistically significant (Lee et al. ${ }^{6)}$ study in 2011, SMD=-0.386; NCT00938704 ${ }^{17)}$ in 2011, $\mathrm{SMD}=-0.140$; Brignole 2005, $\mathrm{SMD}=-0.536$ ). In contrast, the final study significantly favored CMC (Sanchez et al. ${ }^{15)}$ study in 2010, SMD=-1.665; $95 \%$ confidence interval [CI], -2.527 to -0.803$)$.

Study

ID

\begin{tabular}{lll} 
Baudouin et al. ${ }^{11)}(2012)$ & $-0.01(-0.49$ to 0.48$)$ & 23.35 \\
Lee et al. ${ }^{6)}(2011)$ & $-0.39(-0.88$ to 0.11$)$ & 23.23 \\
NCT00938704 ${ }^{17)}(2011)$ & $-0.14(-0.61$ to 0.33$)$ & 23.76 \\
Sanchez et al. ${ }^{15)}(2010)$ & $-1.66(-2.53$ to -0.80$)$ & 14.95 \\
Brignole et al. ${ }^{13)}(2005)$ & $-0.54(-1.41$ to 0.34$)$ & 14.71 \\
Overall $\left(I^{2}=66.1 \%, \mathrm{P}=0.019\right)$ & $-0.45(-0.91$ to 0.01$)$ & 100.00 \\
\hline & 0 & Favours HA
\end{tabular}

\section{Meta-Analysis Results}

In terms of mean TBUT change from baseline and its standard deviation, we conducted continuous variable meta-analysis. The Hedges random effect model analysis showed that the overall pooled SMD of TBUT was -0.452 (95\% CI, -0.911 to 0.007), as shown in Figure 2. There was no significant difference between CMC and HA groups (P-value $=0.053$ ). The generic inverse variance fixed effect model analysis showed that the overall pooled SMD of TBUT was - 0.334 (95\% CI, -0.588 to -0.081 ), as shown in Figure 3. The CMC group showed significantly more improvement than the HA group (P-value $=0.010$ ).

The heterogeneity $\mathrm{I}^{2}$ among the studies was $66.1 \%$ (P-value=0.019). Accordingly, we conducted additional analysis excluding Sanchez et al. ${ }^{15)}$ in 2010. Meta-analysis of the remaining four studies showed a pooled SMD of -0.208 ( $95 \% \mathrm{CI},-0.473$ to -0.057 ; P-value $=0.124)$, heterogeneity $\mathrm{I}^{2} 0 \%$ (P-value $\left.=0.617\right)$. This result indicated that the existing heterogeneity was established by Sanchez et al. ${ }^{15)}$ study in 2010.
Figure 2.Forest plot of the efficacy of CMC and $\mathrm{HA}$ (random effect model). CMC, carboxymethylcelluose; $\mathrm{HA}$, hyaluronate.

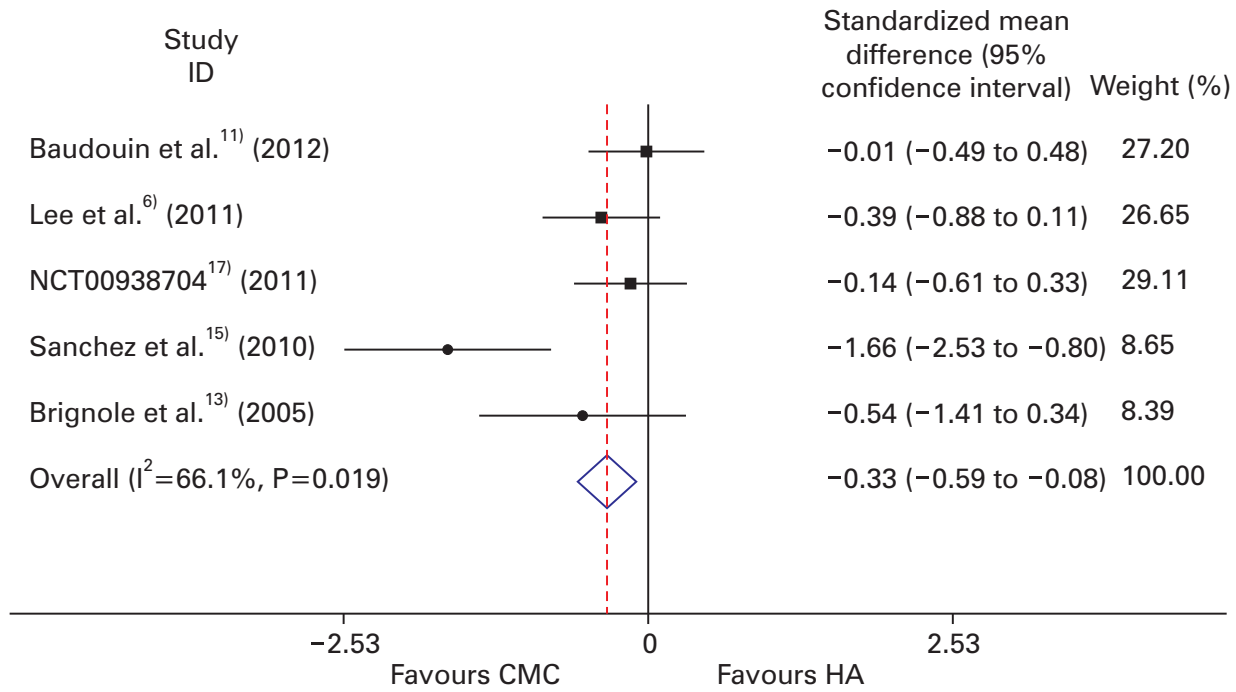

Figure 3. Forest plot of the efficacy of CMC and $\mathrm{HA}$ (fixed effect model). CMC, carboxymethylcelluose; HA, hyaluronate. 


\section{Risk of Bias}

The risk of bias in the five RCTs was low (Figure 4). However, the domain of blinding of participants and personnel was ranked high as four studies were designed as observer-masked single-blind trials; only the NCT00938704 ${ }^{17)}$ study in 2011 was a double-blind trial. Incomplete data were found in the studies of both Sanchez et al. ${ }^{15)}$ study in 2010 and Brignole et al. ${ }^{13)}$ study in 2005, and the missing values were replaced by estimates using adequate statistical techniques. Finally, although Sanchez et al. ${ }^{15)}$ study in 2010 was a RCT, the mean age of the study population between the two groups was significantly different. It was therefore ranked unclear for the domain of other bias.

\section{DISCUSSION}

This study found that CMC was more efficacious than HA in treating dry eye disease. Due to the anionic characteristic of CMC, the lengthened tear retention time could influence the tear film stability, ${ }^{6}$ leading to the reduction of tear film hyperosmolarity, and possible inflammation alleviation. Indeed, Sanchez et al. ${ }^{15)}$ study in 2010 revealed a greater reduction of inflammatory markers in the CMC group. However, the studies did show contradictory results. Brignole et al. ${ }^{13)}$ study in 2005 found that HA decreased ocular surface inflammation more than CMC, although not significantly. The greater water retention capacity

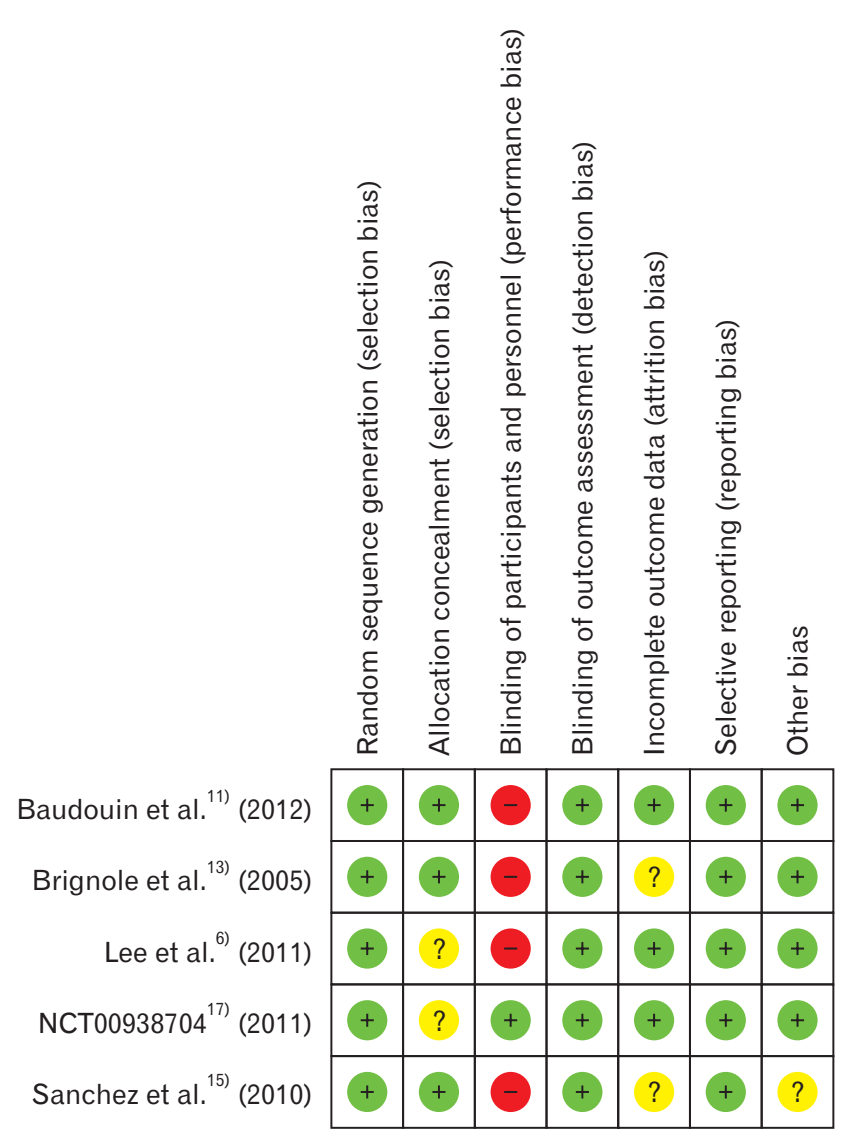

Figure 4. Risk of bias summary. of HA may improve the epithelial repair process. ${ }^{5,6)}$ However, it is important to note that the active ingredient of artificial tears is not the only factor that determines the effect on dry eye disease. Other elements, such as mineral composition or final concentration, may influence the results. In the case of HA eye drops, while there was a broad concentration range $(0.1 \%$ to $3 \%)$, low-concentration eye drops were commonly used in clinical settings. Indeed, all the included studies used low-concentration products.

Results might also be affected by drug compliance. Baudouin et al. ${ }^{11)}$ study in 2012 reported that patients were more satisfied with CMC eye drops, because they were applied less frequently than HA. Additionally, patients tended to prefer a multi-dose bottled preparation as unitdose artificial tears were more costly and less convenient to use. ${ }^{11)}$ However, multi-dose eye drops, which contained preservatives, might cause toxic or allergic reactions when applied frequently. ${ }^{22)}$ These factors may have significantly affected patient compliance and effectiveness over long-term treatment. ${ }^{11)}$

Many studies addressed various methods to evaluate dry eye disease, such as TBUT, corneal or conjunctival staining, Schirmer test, and dry eye symptom score. While these tests are all objective tests, we focused on TBUT as it was the measurement most suitable for comparison. Although most studies evaluated corneal or conjunctival staining and dry eye symptom score, their scale or index was inconsistent across screened studies. While these showed similar tendencies in each study, the staining and symptom score results could not be combined.

In our study, effect sizes were measured in two meta-analysis models. Considering that all the studies were consistent RCTs, fixed effect model meta-analysis was performed, which resulted in a significant difference favorable to CMC. However, considering the heterogeneity within the studies, we also conducted random effect model metaanalysis. While the results were also favorable to $\mathrm{CMC}$, they were not statistically significant.

This meta-analysis revealed slightly high heterogeneity, which might originate from differences in the study population and intervention. The Sanchez et al. ${ }^{15)}$ study in 2010 showed a statistical difference in age between the two groups, which may induce differences in the efficacy of treatment. Indeed, only Sanchez et al. ${ }^{15)}$ study in 2010 showed a statistically significant difference between the two treatment groups. In addition, variation in the artificial tear products used might contribute to heterogeneity, namely concentration, mineral composition and the inclusion of preservative ingredients.

The limitations of this review included the paucity of available studies and the small sample sizes of these studies. This limitation was compounded by the use of several tests to diagnose and assess the degree of dry eye disease, decreasing further the available studies for meta-analysis. Therefore, the efficacy of artificial tears might not be fully reflected by TBUT alone.

In conclusion, this systematic review and meta-analysis compared the efficacies of two commonly used artificial tears, CMC and HA, in treating dry eye disease. On the basis of the five studies included in this 
review, our results suggest that the efficacy of CMC is better than that of HA, although not significantly. Further research is needed to better define the differences in efficacy between CMC and HA in treating dry eye disease.

\section{CONFLICT OF INTEREST}

No potential conflict of interest relevant to this article was reported.

\section{REFERENCES}

1. Baudouin C. The pathology of dry eye. Surv Ophthalmol 2001;45 Suppl 2:S211-20.

2. Lemp MA. Report of the National Eye Institute/Industry workshop on Clinical Trials in Dry Eyes. CLAO J 1995;21:221-32.

3. The definition and classification of dry eye disease: report of the Definition and Classification Subcommittee of the International Dry Eye WorkShop (2007). Ocul Surf 2007;5:75-92.

4. Johnson ME, Murphy PJ. Changes in the tear film and ocular surface from dry eye syndrome. Prog Retin Eye Res 2004;23:449-74.

5. Aragona P, Papa V, Micali A, Santocono M, Milazzo G. Long term treatment with sodium hyaluronate-containing artificial tears reduces ocular surface damage in patients with dry eye. Br J Ophthalmol 2002;86:181-4.

6. Lee JH, Ahn HS, Kim EK, Kim TI. Efficacy of sodium hyaluronate and carboxymethylcellulose in treating mild to moderate dry eye disease. Cornea 2011;30:175-9.

7. Hunt G, Kearney P, Kellaway I. Mucoadhesive polymers in drug delivery systems. In: Johnson P, Lloyd-Jones JG; Society for Drug Research, editors. Drug delivery systems: fundamentals and techniques. Chichester: Ellis Horwood; 1987. p. 180-99.

8. Peppas NA, Buri PA. Surface, interfacial and molecular aspects of polymer bioadhesion on soft tissues. J Control Release 1985;2:257-75.

9. Meyer K. Chemical structure of hyaluronic acid. Fed Proc 1958;17:1075-7.

10. Nakamura M, Hikida M, Nakano T, Ito S, Hamano T, Kinoshita S. Characterization of water retentive properties of hyaluronan. Cornea 1993;12:433-6.

11. Baudouin C, Cochener B, Pisella PJ, Girard B, Pouliquen P, Cooper H, et al. Randomized, phase III study comparing osmoprotective carboxymethylcellulose with sodium hyaluronate in dry eye disease. Eur J Ophthalmol 2012;22:751-61.
12. Benelli U, Nardi M, Posarelli C, Albert TG. Tear osmolarity measurement using the TearLab Osmolarity System in the assessment of dry eye treatment effectiveness. Cont Lens Anterior Eye 2010;33:61-7.

13. Brignole F, Pisella PJ, Dupas B, Baeyens V, Baudouin C. Efficacy and safety of $0.18 \%$ sodium hyaluronate in patients with moderate dry eye syndrome and superficial keratitis. Graefes Arch Clin Exp Ophthalmol 2005;243:531-8.

14. Mencucci R, Boccalini C, Caputo R, Favuzza E. Effect of a hyaluronic acid and carboxymethylcellulose ophthalmic solution on ocular comfort and tear-film instability after cataract surgery. J Cataract Refract Surg 2015;41:1699-704.

15. Sanchez MA, Torralbo-Jimenez P, Giron N, de la Heras B, Herrero Vanrell R, Arriola-Villalobos $\mathrm{P}$, et al. Comparative analysis of carmellose $0.5 \%$ versus hyaluronate $0.15 \%$ in dry eye: a flow cytometric study. Cornea 2010;29:167-71.

16. Simmons PA, Liu H, Carlisle-Wilcox C, Vehige JG. Efficacy and safety of two new formulations of artificial tears in subjects with dry eye disease: a 3-month, multicenter, active-controlled, randomized trial. Clin Ophthalmol 2015;9:665-75.

17. NCT00938704. Study to compare the efficacy and safety of two nonpreserved artificial tears for the treatment of dry eye signs and symptoms [Internet]. Bethesda (MD): National Institutes of Health; 2011 [cited 2016 Feb 14]. Available from: https://clinicaltrials.gov/ct2/ show/NCT00938704.

18. NCT00987727. Safety and efficacy of carboxymethylcellulose for ocular surface integrity in symptomatic dry eye [Internet]. Bethesda (MD): National Institutes of Health; 2011 [cited 2016 Feb 14]. Available from: https://clinicaltrials.gov/ct2/show/NCT00987727?term=NCT00987727 \&rank=1.

19. Liberati A, Altman DG, Tetzlaff J, Mulrow C, Gotzsche PC, Ioannidis JP, et al. The PRISMA statement for reporting systematic reviews and meta-analyses of studies that evaluate health care interventions: explanation and elaboration. PLoS Med 2009;6:e1000100.

20. Hedges LV, Olkin I. Statistical methods for meta-analysis. Orlando (FL): Academic Press; 1985.

21. Higgins JP, Green S. Cochrane handbook for systematic reviews of interventions version 5.1.0 [Internet]. London: The Cochrane Collaboration; 2011 [cited 2016 Feb 14]. Available from: http://handbook. cochrane.org/.

22. Furrer P, Mayer JM, Gurny R. Ocular tolerance of preservatives and alternatives. Eur J Pharm Biopharm 2002;53:263-80. 